Article

\title{
Multiple Attribute Decision-Making Method Using Linguistic Cubic Hesitant Variables
}

\author{
Jun Ye* and Wenhua Cui \\ Department of Electrical Engineering and Automation, Shaoxing University, 508 Huancheng West Road, \\ Shaoxing 312000, China; wenhuacui@usx.edu.cn \\ * Correspondence: yejun@usx.edu.cn; Tel.: +86-575-8832-7323
}

Received: 9 August 2018; Accepted: 5 September 2018; Published: 7 September 2018

\begin{abstract}
Linguistic decision making (DM) is an important research topic in DM theory and methods since using linguistic terms for the assessment of the objective world is very fitting for human thinking and expressing habits. However, there is both uncertainty and hesitancy in linguistic arguments in human thinking and judgments of an evaluated object. Nonetheless, the hybrid information regarding both uncertain linguistic arguments and hesitant linguistic arguments cannot be expressed through the various existing linguistic concepts. To reasonably express it, this study presents a linguistic cubic hesitant variable (LCHV) based on the concepts of a linguistic cubic variable and a hesitant fuzzy set, its operational relations, and its linguistic score function for ranking LCHVs. Then, the objective extension method based on the least common multiple number/cardinality for LCHVs and the weighted aggregation operators of LCHVs are proposed to reasonably aggregate LCHV information because existing aggregation operators cannot aggregate LCHVs in which the number of their hesitant components may imply difference. Next, a multi-attribute decision-making (MADM) approach is proposed based on the weighted arithmetic averaging (WAA) and weighted geometric averaging (WGA) operators of LCHVs. Lastly, an illustrative example is provided to indicate the applicability of the proposed approaches.
\end{abstract}

Keywords: linguistic cubic hesitant variable; least common multiple number; weighted aggregation operator; linguistic score function; decision making

\section{Introduction}

Decision making (DM) theory and methods is an important research field [1-4], while linguistic $\mathrm{DM}$ is a critical topic in DM theory and methods since using linguistic terms and arguments, such as "good" and "very good", for the assessment of the objective world is very fitting for human thinking and expressing habits. In particular, human linguistic expression has precedence over the numerical value expression regarding qualitative attributes in the DM process. Firstly, the concept of a linguistic variable (LV) was presented for its approximate reasoning application [5]. Then, a linguistic DM problem was solved using linguistic arguments [6,7]. Next, various linguistic aggregation operators were introduced for (group) DM problems [8-12]. In the interval/uncertain linguistic setting, various aggregation operators of interval/uncertain LVs were presented for uncertain linguistic DM problems [13-17]. Under determinate and indeterminate linguistic situations, the linguistic cubic variable (LCV) that consists of its interval/uncertain LV and its certain LV was presented as the linguistic extension of a cubic set in [18] and the weighted aggregation operators of LCVs $[19,20]$ were proposed for DM problems with LCV information. On the one hand, a neutrosophic linguistic number (NLN), which is considered as a changeable interval linguistic number depending on its indeterminacy, and the weighted aggregation operators of NLNs were introduced for group DM problems with NLN information [21], and then the linguistic neutrosophic uncertain number 
(LNUN) that consists of the truth, falsity, and indeterminacy NLNs, and its weighted aggregation operators was introduced for group DM problems with LNUN information [22]. On the other hand, the linguistic neutrosophic variable/number (LNV/LNN) that consists of the truth, falsity, and indeterminacy LVs and the aggregation operators of LNVs [23-25] was presented, and then the correlation coefficients and cosine measures of LNVs $[26,27]$ were introduced for (group) DM problems under truth, falsity, and indeterminacy linguistic environments. Based on the hybrid idea of both an interval LNV and a single-valued LNV, a linguistic neutrosophic cubic variable (LNCV) and some aggregation operators of LNCVs $[28,29]$ were proposed for DM problems with LNCV information. Furthermore, the single-valued linguistic neutrosophic interval linguistic number (SVLNILN) that is composed of both its interval LV (its uncertain linguistic argument) and its single-valued LNV (its confident argument) and the weighted aggregation operators of SVLNILNs were proposed for DM problems with SVLNILN information [30]. Regarding decision makers' hesitancy in the NLN DM process, the expected value and similarity measure of hesitant NLNs based on the objective extension method of the least common multiple number/cardinality (LCMN/LCMC) [31] were introduced for multi-attribute DM (MADM) problems with hesitant NLN information. Furthermore, the similarity measures of hesitant LNVs based on the LCMN extension method [32] were presented for MADM problems with hesitant LNN information.

However, DM information composed of an interval linguistic value (an uncertain linguistic part) and a hesitant linguistic set (HLS) (a hesitant linguistic part) exists in some DM problems due to decision makers' uncertainty and hesitancy regarding an evaluated object. For instance, when we require five experts to assess the service quality of a hotel from the linguistic term set (LTS) $Y$ $=\left\{y_{0}\right.$ (extremely poor), $y_{1}$ (very poor), $y_{2}$ (poor), $y_{3}$ (slightly poor), $y_{4}$ (moderate), $y_{5}$ (goodish), $y_{6}$ (good), $y_{7}$ (very good), $y_{8}$ (extremely good)\}, the interval linguistic value $\left[y_{5}, y_{7}\right]$ is given by two of the five experts and the HLS $\left\{y_{4}, y_{5}, y_{6}\right\}$ is given by three of the five experts under the situation of their uncertainty and hesitancy, and then the hybrid form of both $\left[y_{5}, y_{7}\right]$ (the uncertain linguistic part) and $\left\{y_{4}, y_{5}, y_{6}\right\}$ (the hesitant linguistic part) cannot be expressed simultaneously using the aforementioned various linguistic concepts. Clearly, this expression problem requires us to solve the gap using a hybrid linguistic form. Hence, this study presents a new linguistic concept based on the combining form of both an LCV and a hesitant LV, which is called a linguistic cubic hesitant variable (LCHV), the operations and ranking method of LCHVs so as to solve MADM problems under the situation of decision makers' uncertainty and hesitancy. In this study framework, Section 2 presents a LCHV concept to express the hybrid information of both an interval linguistic argument and a hesitant linguistic argument, and then the operational relations and linguistic score function of LCHVs. Section 3 presents the WAA and WGA operators of LCHVs based on the LCMN extension method, which contain the objectivity and suitability of the aggregation operations for LCHVs, and discusses their properties. Next, a MADM approach is proposed based on both the WAA and the WGA operators of LCHVs and the linguistic score function of LCHVs in Section 4. Section 5 applies the proposed MADM approach to the MADM problem regarding an illustrative example in a LCHV setting, and then its decision results show its applicability. Lastly, conclusions and the next study are contained in Section 6.

\section{Linguistic Cubic Hesitant Variables (LCHVs)}

This section presents the concept of the LCHV, along with internal and external LCHV concepts, the linguistic score function of the LCHV for ranking LCHVs, and the operational relations of LCHVs.

Based on the hybrid idea of both an interval/uncertain LV and a hesitant LV, we propose the concept of LCHV, including an internal LCHV and an external LCHV, as below.

Definition 1. Set a LTS as $Y=\left\{y_{l} \mid l \in[0, q]\right\}$, where $q$ is an even number. A LCHV $z$ in $Y$ is constructed using $z=\left(\widetilde{y}_{u}, \widetilde{y}_{h}\right)$, where $\widetilde{y}_{u}=\left[y_{\alpha}, y_{\beta}\right]$ for $\beta \geq \alpha$ and $y_{\alpha}, y_{\beta} \in Y$ is an interval/uncertain LV and $\widetilde{y}_{h}=$ 
$\left\{y_{\lambda_{k}} \mid y_{\lambda_{k}} \in Y, k=1,2, \ldots, r\right\}$ is a set of $\mathrm{r}$ possible LVs (i.e., a hesitant linguistic variable (HLV)) ranked in an ascending order.

Especially when $r=1$, LCHV is reduced to LCV introduced in [19].

Definition 2. Let $z=\left(\widetilde{y}_{u}, \widetilde{y}_{h}\right)$ be a LCHV, with $\widetilde{y}_{u}=\left[y_{\alpha}, y_{\beta}\right]$ for $y_{\alpha}, y_{\beta} \in Y$ and $\widetilde{y}_{h}=$ $\left\{y_{\lambda_{k}} \mid y_{\lambda_{k}} \in Y, k=1,2, \ldots, r\right\}$. Then, we call

(i) $z=\left(\widetilde{y}_{u}, \widetilde{y}_{h}\right)=\left(\left[y_{\alpha}, y_{\beta}\right],\left\{y_{\lambda_{1}}, y_{\lambda_{2}}, \ldots, y_{\lambda_{r}}\right\}\right)$ an internal LCHV if every $\lambda_{k} \in[\alpha, \beta](k=1,2, \ldots, r)$ for $\alpha, \beta \in[0, q]$;

(ii) $z=\left(\widetilde{y}_{u}, \widetilde{y}_{h}\right)=\left(\left[y_{\alpha}, y_{\beta}\right],\left\{y_{\lambda_{1}}, y_{\lambda_{2}}, \ldots, y_{\lambda_{r}}\right\}\right)$ an external LCHV if every $\lambda_{k} \notin[\alpha, \beta](k=1,2, \ldots, r)$ for $\alpha, \beta, \lambda_{k} \in[0, q]$.

For the two LCHVs, $z_{1}=\left(\widetilde{y}_{u 1}, \widetilde{y}_{h 1}\right)$ and $z_{2}=\left(\widetilde{y}_{u 2}, \widetilde{y}_{h 2}\right)$, the number of LVs in $\widetilde{y}_{h 1}$ and $\widetilde{y}_{h 2}$ may imply difference. To realize the suitable operations of different LCHVs, we can extend the two HLVs $\widetilde{y}_{h 1}$ and $\widetilde{y}_{h 2}$ until both reach the same number of LVs on the basis of the LCMN extension method, which shows its objective advantage instead of the subjective extension methods depending on personal preference $[31,32]$.

Suppose $z_{j}=\left(\widetilde{y}_{u j}, \widetilde{y}_{h j}\right)=\left(\left[y_{\alpha_{j}}, y_{\beta_{j}}\right],\left\{y_{\lambda_{j 1}}, y_{\lambda_{j 2}}, \ldots, y_{\lambda_{j r_{j}}}\right\}\right)(j=1,2, \ldots, n)$ is a group of LCHVs and the LCMN of $\left(r_{1}, r_{1}, \ldots, r_{n}\right)$ for $\widetilde{y}_{h j}(j=1,2, \ldots, n)$ is $c$. Then, one can extend them to the same number of LVs based on the following extension forms:

$$
\begin{aligned}
& z_{1}^{e}=\left(\left[y_{\alpha_{1}}, y_{\beta_{1}}\right],\left\{y_{\lambda_{11}^{1}}, y_{\lambda_{11}^{2}}, \ldots y_{\lambda_{11}^{c / r_{1}}}, y_{\lambda_{12}^{1}}, y_{\lambda_{12}^{2}}, \ldots, y_{\lambda_{12}^{c / r_{1}}}, \ldots, y_{\lambda_{1 r_{1}}^{1}}, y_{\lambda_{1 r_{1}}^{2}}, \ldots, y_{\lambda_{1 r_{1}}^{c / r_{1}}}\right\}\right), \\
& z_{2}^{e}=\left(\left[y_{\alpha_{2}}, y_{\beta_{2}}\right],\left\{y_{\lambda_{21}^{1}}, y_{\lambda_{21}^{2}}, \ldots y_{\lambda_{21}^{c / r_{2}}}, y_{\lambda_{22}^{1}}, y_{\lambda_{22}^{2}}, \ldots, y_{\lambda_{22}^{c / r_{2}}}, \ldots, y_{\lambda_{2 r_{2}}^{1},} y_{\lambda_{2 r_{2}}^{2}}, \ldots, y_{\lambda_{2 r_{2}}^{c / r_{2}}}\right\}\right), \\
& z_{n}^{e}=\left(\left[y_{\alpha_{n}}, y_{\beta_{n}}\right],\left\{y_{\lambda_{n 1}^{1}}, y_{\lambda_{n 1}^{2}}, \ldots y_{\lambda_{n 1}^{c / r_{n}}}, y_{\lambda_{n 2}^{1}}, y_{\lambda_{n 2}^{2}}, \ldots, y_{\lambda_{n 2}^{c / r_{n}}}, \ldots, y_{\lambda_{n r_{n}}^{1}}, y_{\lambda_{n r_{n}}^{2}}, \ldots, y_{\lambda_{n r_{n}}^{c / r_{n}}}\right\}\right) .
\end{aligned}
$$

Example 1. Let us consider that $z_{1}=\left(\left[y_{4}, y_{6}\right],\left\{y_{4}, y_{5}\right\}\right)$ and $z_{2}=\left(\left[y_{5}, y_{6}\right],\left\{y_{4}, y_{5}, y_{6}\right\}\right)$ are two linguistic cubic hesitant numbers (LCHNs) in the LTS $Y=\left\{y_{0}, y_{1}, y_{2}, \ldots, y_{8}\right\}$.

Then, the LCMN obtained from $r_{1}=2$ and $r_{2}=3$ in $z_{1}$ and $z_{2}$ is $c=6$. Through Equation (1), the two LCHNs $z_{1}$ and $z_{2}$ can be extended to the following forms:

$$
z_{1}^{e}=\left(\left[y_{4}, y_{6}\right],\left\{y_{4}, y_{4}, y_{4}, y_{5}, y_{5}, y_{5}\right\}\right) \text { and } z_{2}^{e}=\left(\left[y_{5}, y_{6}\right],\left\{y_{4}, y_{4}, y_{5}, y_{5}, y_{6}, y_{6}\right\}\right)
$$

Definition 3. Let $z_{1}=\left(\widetilde{y}_{u 1}, \widetilde{y}_{h 1}\right)=\left(\left[y_{\alpha_{1}}, y_{\beta_{1}}\right],\left\{y_{\lambda_{11}}, y_{\lambda_{12}}, \ldots, y_{\lambda_{1 r}}\right\}\right)$ and $z_{2}=\left(\widetilde{y}_{u 2}, \widetilde{y}_{h 2}\right)=$ $\left(\left[y_{\alpha_{2}}, y_{\beta_{2}}\right],\left\{y_{\lambda_{21}}, y_{\lambda_{22}}, \ldots, y_{\lambda_{2 r}}\right\}\right)$ be two LCHVs in the LTS $Y=\left\{y_{l} \mid l \in[0, q]\right\}$. Then, we define

(i) $z_{1}=z_{2} \Leftrightarrow \widetilde{y}_{u 1}=\widetilde{y}_{u 2}$ and $\widetilde{y}_{h 1}=\widetilde{y}_{h 2}$, i.e., $y_{\alpha_{1}}=y_{\alpha_{2}}, y_{\beta_{1}}=y_{\beta_{2}}$, and $y_{\lambda_{1 k}}=y_{\lambda_{2 k}}$ for $k=1,2, \ldots, r$;

(ii) $z_{1} \subseteq z_{2} \Leftrightarrow \widetilde{y}_{u 1} \subseteq \widetilde{y}_{u 2}$ and $\widetilde{y}_{h 1} \subseteq \widetilde{y}_{h 2}$, i.e., $y_{\alpha_{1}} \leq y_{\alpha_{2}}, y_{\beta_{1}} \leq y_{\beta_{2}}$, and $y_{\lambda_{1 k}} \leq y_{\lambda_{2 k}}$ for $k=1,2, \ldots, r$.

For a convenient comparison in a linguistic setting, we present the linguistic score function of the LCHV and the ranking method of the LCHVs below.

Definition 4. Let $z=\left(\left[y_{\alpha}, y_{\beta}\right],\left\{y_{\lambda_{1}}, y_{\lambda_{2}}, \ldots, y_{\lambda_{r}}\right\}\right)$ be a LCHV in the LTS $Y=\left\{y_{l} \mid l \in[0, q]\right\}$. Then, its linguistic score function is defined as the following:

$$
y_{L(z)}=y_{\frac{1}{2}\left(\frac{1}{2}(\alpha+\beta)+\frac{1}{r} \sum_{k=1}^{r} \lambda_{k}\right)} \text { for } L(z) \in[0, q] .
$$


Definition 5. Let $z_{1}=\left(\left[y_{\alpha_{1}}, y_{\beta_{1}}\right],\left\{y_{\lambda_{11}}, y_{\lambda_{12}}, \ldots, y_{\lambda_{1 r}}\right\}\right)$ and $z_{2}=\left(\left[y_{\alpha_{2}}, y_{\beta_{2}}\right],\left\{y_{\lambda_{21}}, y_{\lambda_{22}}, \ldots, y_{\lambda_{2 r}}\right\}\right)$ be two LCHVs in the LTS $Y=\left\{y_{l} \mid l \in[0, q]\right\}$. Then, their linguistic scores are $y_{L\left(z_{1}\right)}$ and $y_{L\left(z_{2}\right)}$. Thus, the ranking relations are defined as follows:

(a) If $y_{L\left(z_{1}\right)}>y_{L\left(z_{2}\right)}$, then $z_{1}>z_{2}$;

(b) If $y_{L\left(z_{1}\right)}<y_{L\left(z_{2}\right)}$, then $z_{1}<z_{2}$;

(c) If $y_{L\left(z_{1}\right)}=y_{L\left(z_{2}\right)}$, then $z_{1}=z_{2}$.

Example 2. Let us consider that $z_{1}=\left(\left[y_{5}, y_{6}\right],\left\{y_{4}, y_{6}\right\}\right)$ and $z_{2}=\left(\left[y_{4}, y_{6}\right],\left\{y_{3}, y_{5}, y_{6}\right\}\right)$ are two LCHNs in the LTS $Y=\left\{y_{0}, y_{1}, y_{2}, \ldots, y_{8}\right\}$.

Then, through Equation (2), we calculate the linguistic score values of the two LCHNs below:

$$
\begin{gathered}
y_{L\left(z_{1}\right)}=y_{\frac{1}{2}\left(\frac{1}{2}\left(\alpha_{1}+\beta_{1}\right)+\frac{1}{2} \sum_{k=1}^{2} \lambda_{1 k}\right)}=y_{\frac{1}{2}\left(\frac{1}{2}(5+6)+\frac{1}{2}(4+6)\right)}=y_{5.25} \\
y_{L\left(z_{2}\right)}=y_{\frac{1}{2}\left(\frac{1}{2}\left(\alpha_{2}+\beta_{2}\right)+\frac{1}{3} \sum_{k=1}^{3} \lambda_{2 k}\right)}=y_{\frac{1}{2}\left(\frac{1}{2}(4+6)+\frac{1}{3}(3+5+6)\right)}=y_{4.8333}
\end{gathered}
$$

Since there is $y_{L\left(z_{1}\right)}>y_{L\left(z_{2}\right)}$, they are ranked as $z_{1}>z_{2}$.

Definition 6. Let $z_{1}=\left(\left[y_{\alpha_{1}}, y_{\beta_{1}}\right],\left\{y_{\lambda_{11}}, y_{\lambda_{12}}, \ldots, y_{\lambda_{1 r}}\right\}\right)$ and $z_{2}=\left(\left[y_{\alpha_{2}}, y_{\beta_{2}}\right],\left\{y_{\lambda_{21}}, y_{\lambda_{22}}, \ldots, y_{\lambda_{2 r}}\right\}\right)$ be two LCHVs in the LTS $Y=\left\{y_{l} \mid l \in[0, q]\right\}$. Then, their operational relations are defined as below:

$$
\begin{aligned}
& z_{1}+z_{2}=\left(\left[y_{\alpha_{1}}, y_{\beta_{1}}\right],\left\{y_{\lambda_{11}}, y_{\lambda_{12}}, \ldots, y_{\lambda_{1 r}}\right\}\right)+\left(\left[y_{\alpha_{2}}, y_{\beta_{2}}\right],\left\{y_{\lambda_{21}}, y_{\lambda_{22}}, \ldots, y_{\lambda_{2 r}}\right\}\right)
\end{aligned}
$$

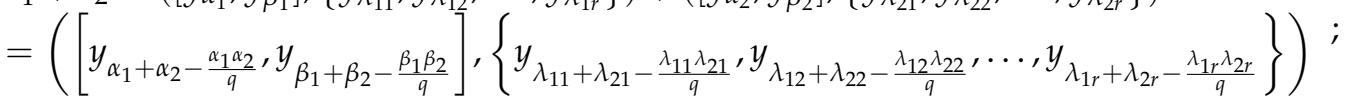

$$
\begin{aligned}
& z_{1} \times z_{2}=\left(\left[y_{\alpha_{1}}, y_{\beta_{1}}\right],\left\{y_{\lambda_{11}}, y_{\lambda_{12}}, \ldots, y_{\lambda_{1 r}}\right\}\right) \times\left(\left[y_{\alpha_{2}}, y_{\beta_{2}}\right],\left\{y_{\lambda_{21}}, y_{\lambda_{22}}, \ldots, y_{\lambda_{2 r}}\right\}\right) \\
& =\left(\left[y_{\frac{\alpha_{1} \alpha_{2}}{q}}, y_{\frac{\beta_{1} \beta_{2}}{q}}\right],\left\{y_{\frac{\lambda_{11} \lambda_{21}}{q}}, y_{\frac{\lambda_{12} \lambda_{22}}{q}}, \ldots, y_{\frac{\lambda_{1 r} \lambda_{2 r}}{q}}\right\}\right)^{2} \\
& \delta z_{1}=\delta\left(\left[y_{\alpha_{1}}, y_{\beta_{1}}\right],\left\{y_{\lambda_{11}}, y_{\lambda_{12}}, \ldots, y_{\lambda_{1 r}}\right\}\right)
\end{aligned}
$$

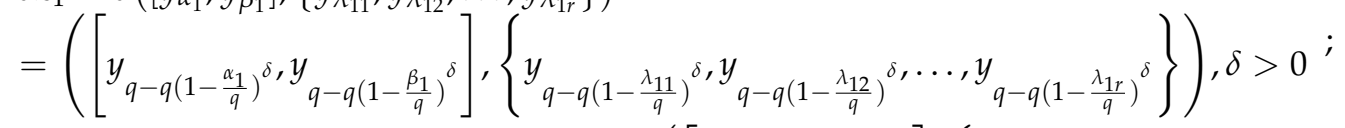

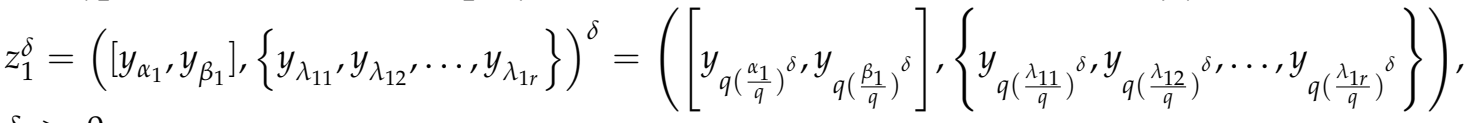

$$
\begin{aligned}
& \delta>0
\end{aligned}
$$

It is obvious that the above calculational results are still LCHVs.

\section{Weighted Aggregation Operators of LCHVs}

\subsection{Weighted Arithmetic Averaging (WAA) Operator of LCHVs}

Definition 7. Set $z_{j}=\left(\left[y_{\alpha_{j}}, y_{\beta_{j}}\right],\left\{y_{\lambda_{j 1}}, y_{\lambda_{j 2}}, \ldots, y_{\lambda_{j r}}\right\}\right)(j=1,2, \ldots, n)$ as a group of LCHVs in $Y=\left\{y_{l} \mid l \in\right.$ $[0, q]\}$, along with its weight $\omega_{j} \in[0,1]$ for $\sum_{j=1}^{n} \omega_{j}=1$. The corresponding WAA operator of the LCHVs is expressed using

$$
F_{L C H V}\left(z_{1}, z_{2}, \cdots, z_{n}\right)=\sum_{j=1}^{n} \omega_{j} z_{j}
$$

Based on Definitions 6 and 7, there exists the following theorem: 
Theorem 1. Set $z_{j}=\left(\left[y_{\alpha_{j}}, y_{\beta_{j}}\right],\left\{y_{\lambda_{j 1}}, y_{\lambda_{j 2}}, \ldots, y_{\lambda_{j r}}\right\}\right)(j=1,2, \ldots, n)$ as a group of LCHVs in $Y=\left\{y_{l} \mid l \in\right.$ $[0, q]\}$, along with its weight $\omega_{j} \in[0,1]$ for $\sum_{j=1}^{n} \omega_{j}=1$, then the aggregation result of Equation (3) is still a $L C H V$, which is calculated using the following aggregation operation:

$$
\begin{aligned}
& F_{L C H V}\left(z_{1}, z_{2}, \cdots, z_{n}\right)=\sum_{j=1}^{n} \omega_{j} z_{j}
\end{aligned}
$$

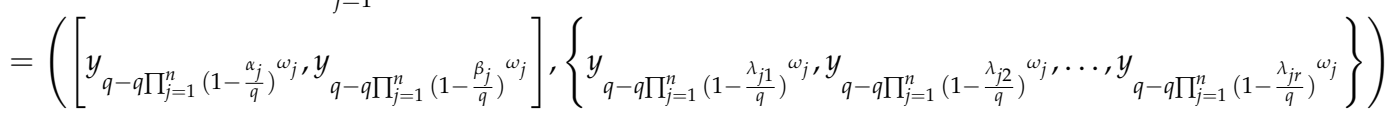

Then, Theorem 1 can be proved based on the mathematical induction below.

\section{Proof.}

(1) Set $n=2$, based on the operational relation (3) in Definition 6, we can get

$$
\begin{aligned}
& \omega_{1} z_{1}=\left(\left[y_{q-q\left(1-\frac{\alpha_{1}}{q}\right)} \omega_{1}, y_{q-q\left(1-\frac{\beta_{1}}{q}\right)} \omega_{1}\right],\left\{y_{q-q\left(1-\frac{\lambda_{11}}{q}\right)} \omega_{1}, y_{\left.\left.q-q\left(1-\frac{\lambda_{12}}{q}\right)^{\omega_{1}}, \ldots, y_{q-q\left(1-\frac{\lambda_{1 r}}{q}\right)}{ }^{\omega_{1}}\right\}\right)}\right.\right. \\
& \omega_{2} z_{2}=\left(\left[y_{q-q\left(1-\frac{\alpha_{2}}{q}\right)} \omega_{2}, y_{q-q\left(1-\frac{\beta_{2}}{q}\right)} \omega_{2}\right],\left\{y_{q-q\left(1-\frac{\lambda_{21}}{q}\right)} \omega_{2}, y_{q-q\left(1-\frac{\lambda_{22}}{q}\right)} \omega_{2}, \ldots, y_{q-q\left(1-\frac{\lambda_{2 r}}{q}\right)} \omega_{2}\right\}\right)
\end{aligned}
$$

Through Equation (3) and the operational relation (1) in Definition 6, their weighted aggregation result is given as

$$
\begin{aligned}
& F_{L C H V}\left(z_{1}, z_{2}\right)=\omega_{1} z_{1}+\omega_{2} z_{2}
\end{aligned}
$$

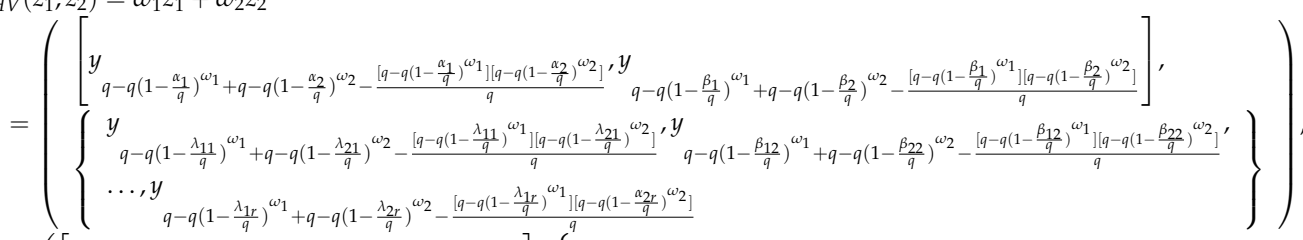

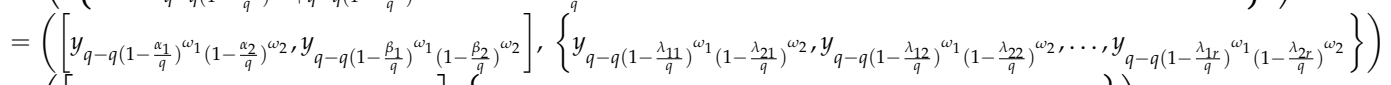

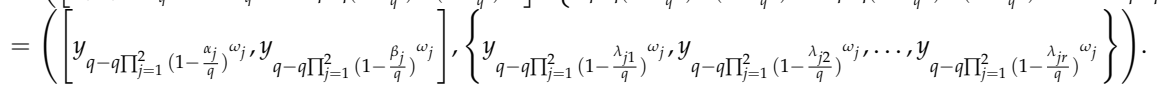

(2) Set $n=k$, the aggregation result of LCHVs based on Equation (4) can be expressed as

$$
\begin{aligned}
& F_{L C H V}\left(z_{1}, z_{2}, \cdots, z_{k}\right)=\sum_{j=1}^{k} \omega_{j} z_{j}
\end{aligned}
$$

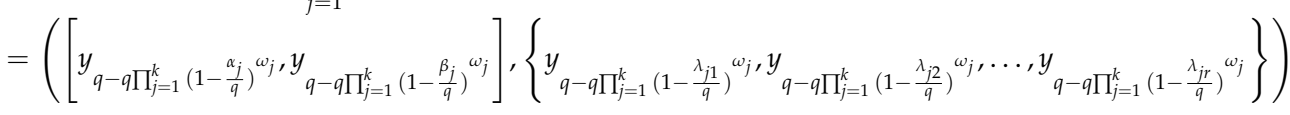

(3) Set $n=k+1$, based on Equations (7) and (8), the aggregation result of the LCHVs is given by

$$
\begin{aligned}
& F_{L C H V}\left(z_{1}, z_{2}, \cdots, z_{k}\right)=\sum_{j=1}^{k} \omega_{j} z_{j}+\omega_{k+1} z_{k+1}
\end{aligned}
$$

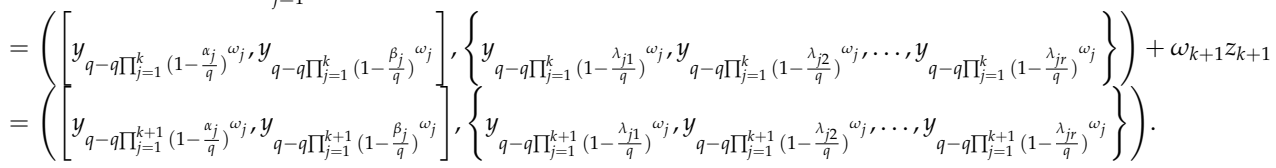

Hence, Equation (4) is valid for any $n$. 
It is noted that we must make the number of linguistic values of each HLS in LCHNs equal based on the LCMN extension method before using Equation (4) in some cases so as to reach the suitable aggregation operation. The following example is given to illustrate the real operational process:

Example 3. Set $z_{1}=\left(\left[y_{5}, y_{6}\right],\left\{y_{4}, y_{6}\right\}\right), z_{2}=\left(\left[y_{4}, y_{6}\right],\left\{y_{3}, y_{5}, y_{6}\right\}\right)$, and $z_{3}=\left(\left[y_{3}, y_{5}\right],\left\{y_{3}, y_{4}, y_{5}\right\}\right)$ as three LCHNs in the LTS $Y=\left\{y_{0}, y_{1}, y_{2}, \ldots, y_{8}\right\}$. Then, their weight vector is given as $\omega=(0.4,0.25,0.35)$.

To reach the suitable aggregation operation by using Equation (4) for the example, we need to extend the three HLSs $\left\{y_{4}, y_{6}\right\},\left\{y_{3}, y_{5}, y_{6}\right\}$ and $\left\{y_{3}, y_{4}, y_{5}\right\}$ into the same components by using the LCMN extension method for them.

First, the LCMN of the three HFSs $\left\{y_{4}, y_{6}\right\},\left\{y_{3}, y_{5}, y_{6}\right\}$ and $\left\{y_{3}, y_{4}, y_{5}\right\}$ is obtained as $c=6$. Thus, their extension forms are expressed as $z_{1}^{e}=\left(\left[y_{5}, y_{6}\right],\left\{y_{4}, y_{4}, y_{4}, y_{6}, y_{6}, y_{6}\right\}\right), z_{2}^{e}=\left(\left[y_{4}, y_{6}\right],\left\{y_{3}, y_{3}, y_{5}, y_{5}, y_{6}, y_{6}\right\}\right)$, and $z_{3}^{e}=\left(\left[y_{3}, y_{5}\right],\left\{y_{3}, y_{3}, y_{4}, y_{4}, y_{5}, y_{5}\right\}\right)$.

Through Equation (4), their operational result of the WAA operator is given by

$$
\begin{aligned}
& F_{L C H V}\left(z_{1}^{e}, z_{2}^{e}, z_{3}^{e}\right)=\sum_{j=1}^{3} \omega_{j} z_{j}^{e}
\end{aligned}
$$

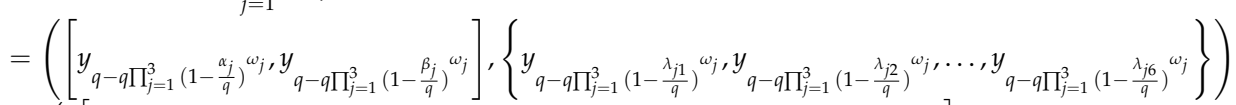

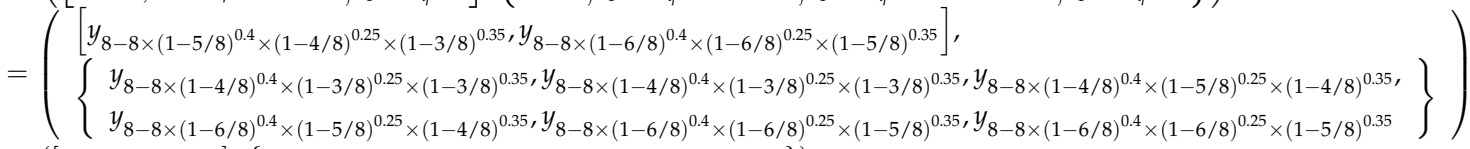

$$
\begin{aligned}
& =\left(\left[y_{4.1452}, y_{5.6950}\right],\left\{y_{3.4269}, y_{3.4269}, y_{4.2776}, y_{5.1789}, y_{5.6950}, y_{5.6950}\right\}\right) \text {. }
\end{aligned}
$$

It is obvious that their aggregated result is still LCHN and all the linguistic values in the aggregated LCHN still belong to $Y$, and then the WAA operator of the LCHNs based on the LCMN extension method shows its objective extension operation without the subjective extension forms depending on decision makers' preferences, which demonstrate the advantage of its operational rationality.

Theorem 2. Set $z_{j}(j=1,2, \ldots, n)$ as a group of LCHVs. Then, the WAA operator of $F_{L C H V}\left(z_{1}, z_{2}, \cdots, z_{n}\right)$ indicates the following properties:

(1) Idempotency: If $z_{j}=z(j=1,2, \ldots, n)$, then there exists $F_{L C H V}\left(z_{1}, z_{2}, \cdots, z_{n}\right)=z$.

(2) Boundedness: Set $z^{+}=\left(\left[\max _{j}\left(y_{\alpha_{j}}\right), \max _{j}\left(y_{\beta_{j}}\right)\right],\left\{\max _{j}\left(y_{\lambda_{j 1}}\right), \max _{j}\left(y_{\lambda_{j 2}}\right), \ldots, \max _{j}\left(y_{\lambda_{j r}}\right)\right\}\right)$ and $z^{-}=\left(\left[\min _{j}\left(y_{\alpha_{j}}\right), \min _{j}\left(y_{\beta_{j}}\right)\right],\left\{\min _{j}\left(y_{\lambda_{j 1}}\right), \min _{j}\left(y_{\lambda_{j 2}}\right), \ldots, \min _{j}\left(y_{\lambda_{j r}}\right)\right\}\right)(j=1,2, \ldots, n)$ as the maximum $L C H V$ and the minimum $L C H V$, respectively. Then, $z^{-} \leq F_{L C H V}\left(z_{1}, z_{2}, \cdots, z_{n}\right) \leq z^{+}$ can hold.

(3) Monotonicity: If $z_{j} \leq z_{j}^{*}(j=1,2, \ldots, n)$, then there exists $F_{L C H V}\left(z_{1}, z_{2}, \cdots, z_{n}\right) \leq$ $F_{L C H V}\left(z_{1}^{*}, z_{2}^{*}, \cdots, z_{n}^{*}\right)$.

\section{Proof.}

(1) Since $z_{j}=z(j=1,2, \ldots, n)$, the WAA aggregation result of LCHVs can be calculated using

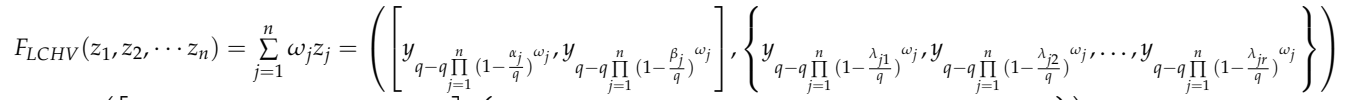

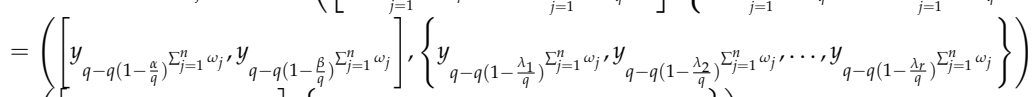

$$
\begin{aligned}
& =\left(\left[y_{q-q\left(1-\frac{\alpha}{q}\right)}, y_{q-q\left(1-\frac{\beta}{q}\right)}\right],\left\{y_{q-q\left(1-\frac{\lambda_{1}}{q}\right)}, y_{q-q\left(1-\frac{\lambda_{2}}{q}\right)}, \ldots, y_{q-q\left(1-\frac{\lambda}{q}\right)}\right\}\right) \\
& =\left(\left[y_{\alpha}, y_{\beta}\right],\left\{y_{\lambda_{1}}, y_{\lambda_{2}}, \ldots, y_{\lambda_{r}}\right\}\right)=z \text {. }
\end{aligned}
$$


(2) Since $z^{-}$and $z^{+}$are the minimum LCHV and the maximum LCHV, respectively, there exists $z^{-} \leq z_{j} \leq z^{+}$. Thus, there is $\sum_{j=1}^{n} \omega_{j} z^{-} \leq \sum_{j=1}^{n} \omega_{j} z_{j} \leq \sum_{j=1}^{n} \omega_{j} z^{+}$. Corresponding to the property (1), there are $\sum_{j=1}^{n} \omega_{j} z^{-}=z^{-}$and $\sum_{j=1}^{n} \omega_{j} z^{+}=z^{+}$. Hence, $z^{-} \leq F_{L C H V}\left(z_{1}, z_{2}, \cdots, z_{n}\right) \leq z^{+}$.

(3) Since $z_{j} \leq z_{j}^{*}(j=1,2, \ldots, n)$, there exists $\sum_{j=1}^{n} \omega_{j} h_{j} \leq \sum_{j=1}^{n} \omega_{j} z_{j}^{*}$. Hence, $F_{L C H V}\left(z_{1}, z_{2}, \cdots, z_{n}\right) \leq$ $F_{L C H V}\left(z_{1}^{*}, z_{2}^{*}, \cdots, z_{n}^{*}\right)$.

Thus, these properties of the WAA operator of LCHVs are proved.

Especially when $\omega_{j}=1 / n(j=1,2, \ldots, n)$, the WAA operator is degenerated to the arithmetic averaging operator of LCHVs. It is obvious that the WAA operator of LCVs in [19] is a special case of the WAA operator of LCHVs when $r=1$.

\subsection{Weighted Geometric Averaging (WGA) Operator of LCHVs}

Definition 8. Set $z_{j}=\left(\left[y_{\alpha_{j}}, y_{\beta_{j}}\right],\left\{y_{\lambda_{j 1}}, y_{\lambda_{j 2}}, \ldots, y_{\lambda_{j r}}\right\}\right)(j=1,2, \ldots, n)$ as a group of LCHVs in $Y=\left\{y_{l} \mid l\right.$ $\in[0, q]\}$, along with its weight $\omega_{j} \in[0,1]$ for $\sum_{j=1}^{n} \omega_{j}=1$. The corresponding WGA operator of LCHVs is defined as

$$
G_{L C H V}\left(z_{1}, z_{2}, \cdots, z_{n}\right)=\prod_{j=1}^{n} z_{j}^{\omega_{j}}
$$

Then, the following theorem can be given based on Definitions 6 and 8 .

Theorem 3. Set $z_{j}=\left(\left[y_{\alpha_{j}}, y_{\beta_{j}}\right],\left\{y_{\lambda_{j 1}}, y_{\lambda_{j 2}}, \ldots, y_{\lambda_{j r}}\right\}\right)(j=1,2, \ldots, n)$ as a group of LCHVs in $Y=\left\{y_{l} \mid l \in\right.$ $[0, q]\}$, along with its weight $\omega_{j} \in[0,1]$ for $\sum_{j=1}^{n} \omega_{j}=1$, then the aggregation result of Equation (11) is still a $\mathrm{LCHV}$, which is computed using the following aggregation operation:

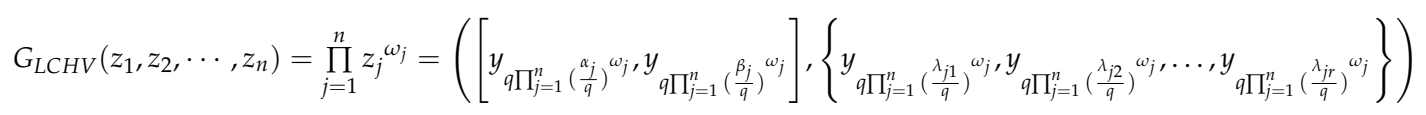

Especially when $\omega_{j}=1 / n(j=1,2, \ldots, n)$, the WGA operator of LCHVs is degenerated to the geometric averaging operator of LCHVs. It is obvious that the WGA operator of LCVs in [19] is also a special case of the WGA operator of LCHVs when $r=1$.

Since the proof of Theorem 3 is similar to that of Theorem 1, it is omitted here.

It is noted that we must make the number of linguistic values of each HLS in LCHNs equal based on the LCMN extension method before using Equation (12) in some cases so as to reach the suitable aggregation operation. The following example is given to illustrate the real operational process:

Example 4. Let us consider Example 3 to compute the aggregation result of the WGA operator of the three LCHNs.

Corresponding to the extension results of the three LCHNs in Example 3, the aggregated result of the three extension LCHNs is given by Equation (12) as follows:

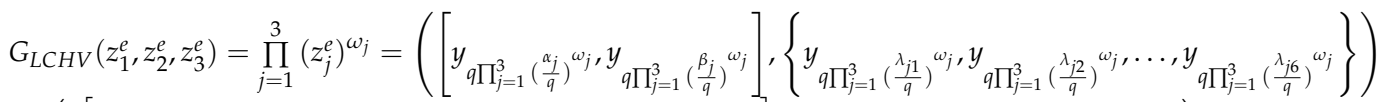

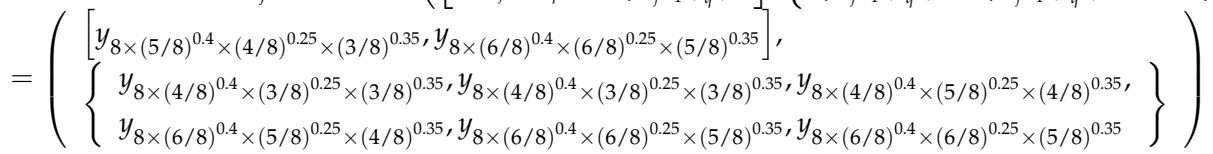

$$
\begin{aligned}
& =\left(\left[y_{3.9545}, y_{5.6291}\right],\left\{y_{3.3659}, y_{3.3659}, y_{4.2295}, y_{4.9742}, y_{5.6291}, y_{5.6291}\right\}\right) \text {. }
\end{aligned}
$$


It is obvious that their aggregated result is still LCHN and all the linguistic values in the aggregated LCHN still belong to $Y$, and the WGA operator of the LCHNs based on the LCMN extension method also shows its objective extension operation without the subjective extension form depending on the decision makers' preferences, which demonstrate the advantage of its operational rationality.

Theorem 4. Set $z_{j}(j=1,2, \ldots, n)$ as a group of LCHVs. Then, the WGA operator of $G_{L C H V}\left(z_{1}, z_{2}, \cdots, z_{n}\right)$ indicates the following properties:

(i) Idempotency: If $z_{j}=z(j=1,2, \ldots, n)$, then there exists $G_{L C H V}\left(z_{1}, z_{2}, \cdots, z_{n}\right)=z$.

(ii) Boundedness: Set $z^{+}=\left(\left[\max _{j}\left(y_{\alpha_{j}}\right), \max _{j}\left(y_{\beta_{j}}\right)\right],\left\{\max _{j}\left(y_{\lambda_{j 1}}\right), \max _{j}\left(y_{\lambda_{j 2}}\right), \ldots, \max _{j}\left(y_{\lambda_{j r}}\right)\right\}\right)$ and $z^{-}=\left(\left[\min _{j}\left(y_{\alpha_{j}}\right), \min _{j}\left(y_{\beta_{j}}\right)\right],\left\{\min _{j}\left(y_{\lambda_{j 1}}\right), \min _{j}\left(y_{\lambda_{j 2}}\right), \ldots, \min _{j}\left(y_{\lambda_{j r}}\right)\right\}\right)(j=1,2, \ldots, n)$ as the maximum LCHV and the minimum $L C H V$, respectively. Then $z^{-} \leq G_{L C H V}\left(z_{1}, z_{2}, \cdots, z_{n}\right) \leq z^{+}$ can hold.

(iii) Monotonicity: If $z_{j} \leq z_{j}^{*}(j=1,2, \ldots, n)$, then there exists $G_{L C H V}\left(z_{1}, z_{2}, \cdots, z_{n}\right) \leq$ $G_{L C H V}\left(z_{1}^{*}, z_{2}^{*}, \cdots, z_{n}^{*}\right)$.

Since the proof of Theorem 4 is similar to that of Theorem 2, it is omitted here.

\section{MADM Approach Using the WAA and WGA Operators of LCHVs}

In this section, a MADM approach is proposed by using the WAA and WGA operators of the LCHVs in a LCHV setting.

For a MADM problem with LCHV information, suppose $Z=\left\{Z_{1}, Z_{2}, \ldots, Z_{m}\right\}$ and $R=\left\{R_{1}, R_{2}, \ldots\right.$, $\left.R_{n}\right\}$ are two sets of $m$ alternatives and $n$ attributes, respectively. When decision makers are requested to assess the alternative $Z_{i}(i=1,2, \ldots, m)$ over the attribute $R_{j}(j=1,2, \ldots, n)$, they may assign an interval linguistic value to $\widetilde{y}_{u i j}$ and a set of several possible linguistic values to $\widetilde{y}_{h i j}$ due to their hesitancy and indeterminacy from the predefined LTS $Y=\left\{y_{l} \mid l \in[0, q]\right\}$, where $q$ is an even number. Thus, the assessed hybrid information of $\widetilde{y}_{u i j}$ and $\widetilde{y}_{h i j}$ corresponding to each attribute $R_{j}$ on each alternative $Z_{i}$ can be represented as a LCHV $z_{i j}=\left(\widetilde{y}_{u i j}, \widetilde{y}_{h i j}\right)=\left(\left[y_{\alpha_{i j}}, y_{\beta_{i j}}\right],\left\{y_{\lambda_{i j(1)}}, y_{\lambda_{i j(2)}}, \ldots, y_{\lambda_{i j\left(r_{i j}\right)}}\right\}\right)$ $(j=1,2, \ldots, n ; i=1,2, \ldots, m)$. Hence, a LCHV decision matrix $M=\left(z_{i j}\right)_{m \times n}$ can be constructed based on all the assessed LCHVs. Then, the weight of each attribute $R_{j}$ is $\omega_{j} \in[0,1]$ and $\sum_{j=1}^{n} \omega_{j}=1$.

Thus, the WAA or WGA operators of the LCHVs and the linguistic score function of the LCHV are utilized to develop a MADM approach with LCHV information, which can be summarized as the following decision steps:

Step 1. The LCMNs of $\left(r_{i 1}, r_{i 2}, \ldots, r_{i n}\right)(i=1,2, \ldots, m)$ in $M=\left(z_{i j}\right)_{m \times n}$ can be obtained as $c_{i}$, where $r_{i j}$ is the number of LVs in $\widetilde{y}_{h i j}$ for $z_{i j}$. Based on the number of occurrences of $c_{i} / r_{i j}$ in a LCHV $z_{i j}$ $(i=1,2, \ldots, m ; j=1,2, \ldots, n), z_{i j}$ is extended to the following form:

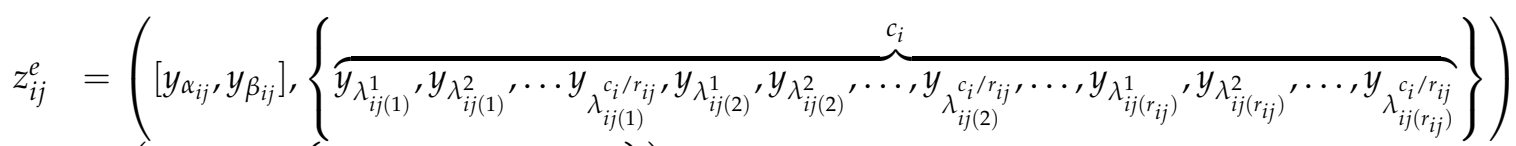

$$
\begin{aligned}
& =\left(\left[y_{\alpha_{i j}}, y_{\beta_{i j}}\right],\left\{y_{\lambda_{i j}^{(1)}}, y_{\lambda_{i j}^{(2)}}, \ldots, y_{\lambda_{i j}^{\left(c_{i j}\right)}}\right\}\right) \text {. }
\end{aligned}
$$

Thus, the extended decision matrix is constructed as

$$
M^{e}=\begin{aligned}
& Z_{1} \\
& Z_{2} \\
& \vdots \\
& Z_{m}
\end{aligned}\left(\begin{array}{llll}
z_{11}^{e} & z_{12}^{e} & \cdots & z_{1 n}^{e} \\
z_{21}^{e} & z_{22}^{e} & \cdots & z_{2 n}^{e} \\
\vdots & \vdots & \vdots & \vdots \\
z_{m 1}^{e} & z_{m 2}^{e} & \cdots & z_{m n}^{e}
\end{array}\right)
$$


Step 2. Based on Equations (4) or (12), the aggregation values of $z_{i}$ for $Z_{i}$ are calculated using the following formula:

$$
\begin{aligned}
& z_{i}=F_{L C H V}\left(z_{i 1}^{e}, z_{i 2}^{e}, \cdots, z_{i n}^{e}\right)=\sum_{j=1}^{n} \omega_{j} z_{i j}^{e}
\end{aligned}
$$

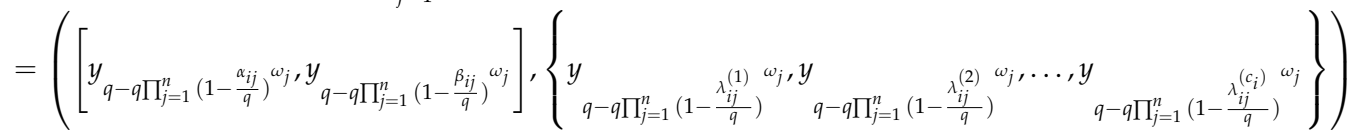

or

$$
\begin{aligned}
& z_{i}=G_{L C H V}\left(z_{i 1}^{e}, z_{i 2}^{e}, \cdots, z_{i n}^{e}\right)=\prod_{j=1}^{n}\left(z_{i j}^{e}\right)^{\omega_{j}}
\end{aligned}
$$

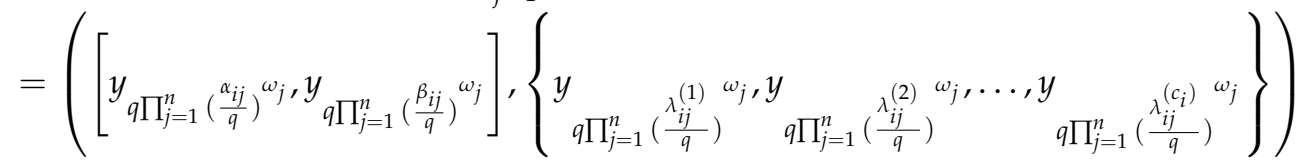

Step 3. The linguistic score values of $y_{L\left(z_{i}\right)}$ for $Z_{i}$ are calculated using Equation (2).

Step 4. All the alternatives are ranked in a descending order of the linguistic score values and the best one is selected corresponding to the biggest linguistic score value.

Step 5. End.

\section{Illustrative Example}

An illustrative example is presented in this section to indicate the application of the proposed MADM approach in a LCHV setting.

A computer company wants to hire a software engineer. Though the human resources department preliminarily selects four potential candidates (alternatives) $-Z_{1}, Z_{2}, Z_{3}$, and $Z_{4}$-from all the applicants, they need to be further assessed based on the requirements (attributes) of innovation capability $\left(R_{1}\right)$, work experience $\left(R_{2}\right)$, and self-confidence $\left(R_{3}\right)$. Then, five experts (decision makers) are invited to choose the most suitable candidate among them in the interview. Here, the importance of the three attributes is indicated by the weight vector $\omega=(0.45,0.35,0.2)$. Thus, the five experts will assess each potential candidate $Z_{\mathrm{i}}(i=1,2,3,4)$ over the three attributes $R_{\mathrm{j}}(j=1,2,3)$ using the hybrid information of uncertain and hesitant linguistic terms so as to express the assessment values of LCHVs from the predefined LTS $Y=\left\{y_{0}\right.$ (extremely poor), $y_{1}$ (very poor), $y_{2}$ (poor), $y_{3}$ (slightly poor), $y_{4}$ (moderate), $y_{5}$ (goodish), $y_{6}$ (good), $y_{7}$ (very good), and $y_{8}$ (extremely good) $\}$ with $q=8$.

For example, in the assessment process of $R_{1}$ for $Z_{1}$, the interval linguistic value $\left[y_{4}, y_{6}\right]$ is assigned by two of the five experts corresponding to the two uncertain ranges $\left[y_{4}, y_{5}\right]$ and $\left[y_{4}, y_{6}\right]$, and then the HLS $\left\{y_{4}, y_{6}\right\}$ is assigned by three of the five experts corresponding to the three linguistic evaluation values $y_{4}, y_{4}$, and $y_{6}$, which can be expressed as the LCHN $\left(\left[y_{4}, y_{6}\right],\left\{y_{4}, y_{6}\right\}\right)$. Using a similar evaluation method, all the LCHNs assessed by the five experts can be constructed as the following LCHN decision matrix:

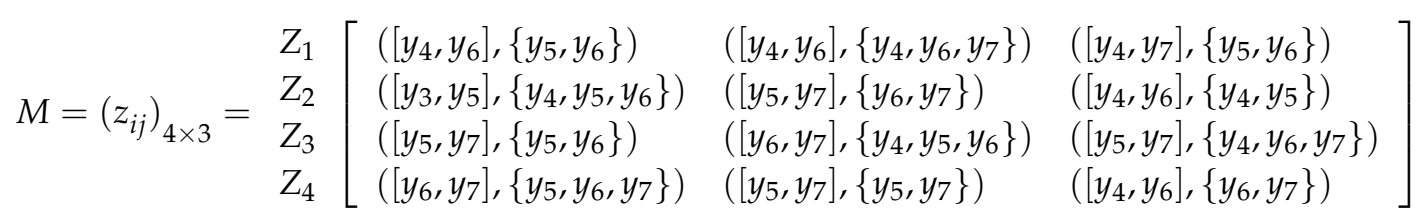

Thus, the MADM approach presented, based on the WAA or WGA operator of the LCHVs, can be utilized for the MADM problem with LCHN information.

For the application, the MADM approach relating to the WAA operator of LCHVs is used for the illustrative example and depicted using the following decision steps: 
Step 1. The LCMNs of $\left(r_{i 1}, r_{i 2}, \ldots, r_{i n}\right)(i=1,2, \ldots, m)$ in $M=\left(z_{i j}\right)_{m \times n}$ can be obtained as $c_{i}=6$ for $i=1,2,3,4$. After that, the decision matrix $M$ can be extended into the extension decision matrix below:

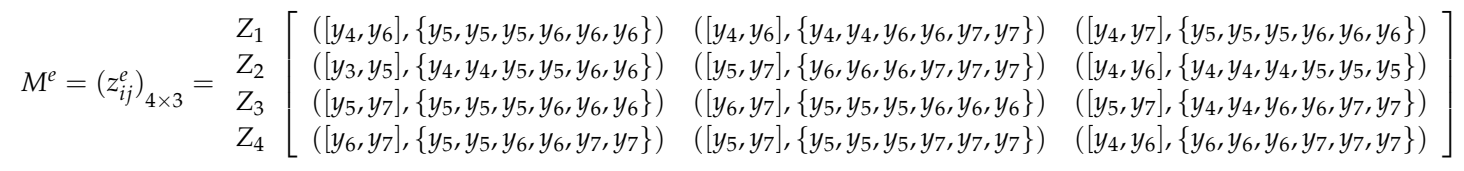

Step 2. Based on Equation (13), the aggregated $L C H N z_{1}$ for $Z_{1}$ can be obtained as follows:

$$
\begin{aligned}
& z_{1}=F_{L C H V}\left(z_{11}^{e}, z_{12}^{e}, z_{13}^{e}\right)=\sum_{j=1}^{3} \omega_{j} z_{1 j}^{e}
\end{aligned}
$$

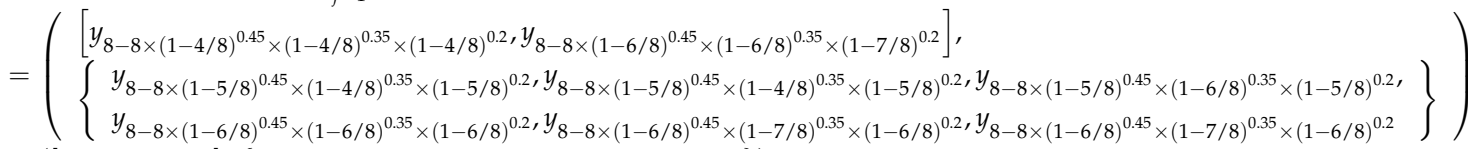

$$
\begin{aligned}
& =\left(\left[y_{4.0000}, y_{6.2589}\right],\left\{y_{4.6822}, y_{4.6822}, y_{5.3969}, y_{6.0000}, y_{6.4308}, y_{6.4308}\right\}\right) \text {. }
\end{aligned}
$$

Using a similar calculation, we can obtain the aggregated LCHNs $z_{i}$ for $Z_{i}(i=2,3,4)$ :

$$
\begin{gathered}
z_{2}=\left(\left[y_{4.0011}, y_{6.1167}\right],\left\{y_{4.8617}, y_{4.8617}, y_{5.2427}, y_{5.9577}, y_{6.2983}, y_{6.2983}\right\}\right), \\
z_{3}=\left(\left[y_{5.3969}, y_{7}\right],\left\{y_{4.8223}, y_{4.8223}, y_{5.2337}, y_{6}, y_{6.2589}, y_{6.2589}\right\}\right), \text { and } \\
z_{4}=\left(\left[y_{5.3523} y_{6.8513}\right],\left\{y_{5.2337} y_{5.2337}, y_{5.695}, y_{6.6340}, y_{7}, y_{7}\right\}\right) .
\end{gathered}
$$

Step 3. Through Equation (2), the linguistic score value of $y_{L\left(z_{1}\right)}$ is given below:

$$
y_{L\left(z_{1}\right)}=y_{\frac{1}{2}\left(\frac{1}{2}\left(\alpha_{1}+\beta_{1}\right)+\frac{1}{6} \sum_{k=1}^{6} \lambda_{1 k}\right)}=y_{\frac{1}{2}\left(\frac{1}{2}(4+6.2589)+\frac{1}{6}(4.6822+4.6822+5.3969+6+6.4308+6.4308)\right)}=y_{5.3666}
$$

Through a similar calculation, we can obtain the linguistic score values of $y_{L\left(z_{i}\right)}(i=2,3,4)$ :

$$
y_{L\left(z_{2}\right)}=y_{5.3228}, y_{L\left(z_{3}\right)}=y_{5.8822}, y_{L\left(z_{4}\right)}=y_{6.1173} \text {. }
$$

Step 4. The four candidates are ranked as $Z_{4} \succ Z_{3} \succ Z_{1} \succ Z_{2}$ based on the linguistic score values. Thus, $Z_{4}$ is the best one among them.

For another application, we can also use the presented MADM method based on the WGA operator of LCHVs for the example and indicate the following decision steps:

Step 1. The same as Step 1.

Step 2. Through Equation (14), the aggregated $\operatorname{LCHN} z_{1}$ for $Z_{1}$ is yielded as follows:

$$
\begin{aligned}
& z_{1}=G_{L C H V}\left(z_{11}^{e}, z_{12}^{e}, z_{13}^{e}\right)=\prod_{j=1}^{3} z_{1 j} \omega_{j}
\end{aligned}
$$

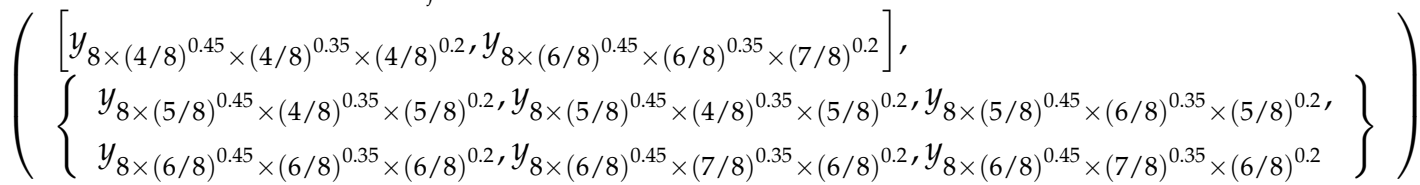

$$
\begin{aligned}
& =\left(\left[y_{4}, y_{6.1879}\right],\left\{y_{4.6244}, y_{4.6244}, y_{5.3295}, y_{6}, y_{6.3326}, y_{6.3326}\right\}\right) \text {. }
\end{aligned}
$$

Using a similar calculation, we can obtain the aggregated LCHNs $z_{i}$ for $Z_{i}(i=2,3,4)$ :

$$
\begin{gathered}
z_{2}=\left(\left[y_{3.7998} y_{5.8338}\right],\left\{y_{4.6099}, y_{4.6099}, y_{5.0968}, y_{5.6249}, y_{6.1059}, y_{6.1059}\right\}\right), \\
z_{3}=\left(\left[y_{5.3295}, y_{7}\right],\left\{y_{4.7818}, y_{4.7818}, y_{5.1857}, y_{6}, y_{6.1879}, y_{6.1879}\right\}\right), \text { and } \\
z_{4}=\left(\left[y_{5.1906}, y_{6.7875}\right],\left\{y_{5.1857}, y_{5.1857}, y_{5.6291} y_{6.5309}, y_{7}, y_{7}\right\}\right) .
\end{gathered}
$$


Step 3. Through Equation (2), the linguistic score value of $y_{L\left(z_{1}\right)}$ is given below:

$$
y_{L\left(\mathrm{z}_{1}\right)}=y_{\frac{1}{2}\left(\frac{1}{2}\left(\alpha_{1}+\beta_{1}\right)+\frac{1}{6} \sum_{k=1}^{6} \lambda_{1 k}\right)}=y_{\frac{1}{2}\left(\frac{1}{2}(4+6.1879)+\frac{1}{6}(4.6244+4.6244+5.3295+6+6.3326+6.3326)\right)}=y_{5.3172}
$$

Using a similar calculation, we can obtain the linguistic score values of $y_{L\left(z_{i}\right)}(i=2,3,4)$ :

$$
y_{L\left(z_{2}\right)}=y_{5.0878,} y_{L\left(z_{3}\right)}=y_{5.8428,} \text { and } y_{L\left(z_{4}\right)}=y_{6.0388 .}
$$

Step 4. The four candidates are ranked as $Z_{4} \succ Z_{3} \succ Z_{1} \succ Z_{2}$. Thus, $Z_{4}$ is still the best one among them.

Clearly, the above two ranking orders based on the WAA and WGA operators of LCHVs and the best candidate are identical in this MADM problem. However, decision makers can use the WAA operator and/or the WGA operators for MADM problems with LCHVs according to their preferences or actual requirements.

Compared with existing MADM methods of LCVs $[19,20]$, the decision information in this study is LCHVs, while the decision information used in References $[19,20]$ is LCVs. As mentioned above, since the LCHV consists of its interval/uncertain LV and its HLV, it is obvious that the LCHV contains more information than the LCV without HLV in References $[19,20]$. However, existing LCV MADM methods in References $[19,20]$ cannot be used to carry out such a DM problem with LCHV information in this paper. Moreover, the proposed MADM approach can solve MADM problems with LCV or LCHV information since LCV is only a special case of a LCHV without the hesitant situation. Therefore, the MADM approach proposed in this study extends the existing one in Reference [19].

Since there is no relative study in existing literature, this is the first time that a new concept of LCHV, the weighted aggregation operators of LCHVs based on the LCMN extension method, and the linguistic score function of LCHV have been proposed in order to solve MADM problems with LCHV information, indicating the main advantages of objectivity and suitability in an indeterminate and hesitant DM setting. However, various existing linguistic DM methods with uncertain or incomplete decision judgments in the realm of MADM cannot carry out the MADM problems with LCHV information because they cannot express and handle the hybrid information of both linguistic cubic arguments and hesitant fuzzy arguments (i.e., LCHV information), which exists in actual DM problems.

\section{Conclusions}

To express the hybrid form of both interval/uncertain linguistic information and hesitant linguistic information, this study originally proposed the LCHV concept, along with the internal LCHV and external LCHV concepts, and the operational relations of LCHVs. Then, the linguistic score function of the LCHV was presented to rank LCHVs. Furthermore, the WAA and WGA operators of LCHVs based on the LCMN extension method were proposed to aggregate LCHV information, and then their properties were indicated. Next, a MADM approach was established based on the WAA and WGA operators of LCHVs to solve MADM problems with LCHV information. Finally, an illustrative example was presented for the application of the developed approach in the LCHN setting. The proposed MADM approach can solve MADM problems with interval/uncertain linguistic and hesitant linguistic arguments in the LCHV setting.

However, the main highlights of this study are summarized below:

(1) The LCHV concept extends the existing LCV concept to express the interval/uncertain linguistic and hesitant linguistic arguments simultaneously for the first time.

(2) This paper proposed two weighted aggregation operators of LCHVs based on the LCMN extension method and the linguistic score function of LCHV for the first time. 
(3) The advantages of the WAA and WGA operators of LCHVs are that: (a) The two aggregation operators based on the LCMN show the objective extension operations without the subjective extension forms depending on decision makers' preferences; (b) all the linguistic values in the aggregated LCHVs still belong to the predefined LTS; and (c) the WAA and WGA operators are the simplest and most common weighted aggregation operations used for MADM problems.

(4) The developed MADM approach with LCHVs extends the existing MADM approaches with LCVs so as to carry out the MADM problems with the hybrid information of both interval linguistic arguments and hesitant linguistic arguments, which the existing ones cannot handle.

Based on this study, new aggregation operators of LCHVs and ranking methods of LCHVs will be further presented and applied in the medical diagnoses of kidney cancer, prostatic cancer, and gastric cancer and the selection problems of suppliers and manufacturing schemes as future work in the LCHV setting.

Author Contributions: J.Y. originally proposed LCHVs, their operations, and the MADM method of LCHVs; W.H.C. provided the calculation and comparative analysis of an illustrative example. The authors wrote the paper together.

Funding: This paper was supported by the National Natural Science Foundation of China (No. 61703280).

Conflicts of Interest: The authors declare no conflicts of interest.

\section{References}

1. Milosavljevic, M.; Bursac, M.; Tričkovic, G. The selection of the railroad container terminal in Serbia based on multi criteria decision making methods. Decis. Mak. Appl. Manag. Eng. 2018, 1, 1-14. [CrossRef]

2. Badi, I.; Ballem, M. Supplier selection using rough BWM-MAIRCA model: A case study in pharmaceutical supplying in Libya. Decis. Mak. Appl. Manag. Eng. 2018, 1, 15-32. [CrossRef]

3. Roy, J.; Adhikary, K.; Kar, S.; Pamučar, D. A rough strength relational DEMATEL model for analysing the key success factors of hospital service quality. Decis. Mak. Appl. Manag. Eng. 2018, 1, 121-142. [CrossRef]

4. Vasiljevic, M.; Fazlollahtabar, H.; Stevic, Z.; Veskovic, S. A rough multicriteria approach for evaluation of the supplier criteria in automotive industry. Decis. Mak. Appl. Manag. Eng. 2018, 1, 82-96. [CrossRef]

5. Zadeh, L.A. The concept of a linguistic variable and its application to approximate reasoning Part I. Inf. Sci. 1975, 8, 199-249. [CrossRef]

6. Herrera, F; Herrera-Viedma, E.; Verdegay, L. A model of consensus in group decision making under linguistic assessments. Fuzzy Sets Syst. 1996, 79, 73-87. [CrossRef]

7. Herrera, F.; Herrera-Viedma, E. Linguistic decision analysis: Steps for solving decision problems under linguistic information. Fuzzy Sets Syst. 2000, 115, 67-82. [CrossRef]

8. $\mathrm{Xu}, \mathrm{Z}$.S. A method based on linguistic aggregation operators for group decision making with linguistic preference relations. Inf. Sci. 2004, 166, 19-30. [CrossRef]

9. $\mathrm{Xu}, \mathrm{Z.S}$. A note on linguistic hybrid arithmetic averaging operator in multiple attribute group decision making with linguistic information. Group Decis. Negot. 2006, 15, 593-604. [CrossRef]

10. Merigó, J.M.; Casanovas, M.; Martínez, L. Linguistic aggregation operators for linguistic decision making based on the Dempster-Shafer theory of evidence. Int. J. Uncertain. Fuzz. Knowl.-Based Syst. 2010, 18, 287-304. [CrossRef]

11. Xu, Y.J.; Merigó, J.M.; Wang, H.M. Linguistic power aggregation operators and their application to multiple attribute group decision making. Appl. Math. Model. 2012, 36, 5427-5444. [CrossRef]

12. Merigó, J.M.; Casanovas, M.; Palacios-Marqués, D. Linguistic group decision making with induced aggregation operators and probabilistic information. Appl. Soft Comput. 2014, 24, 669-678. [CrossRef]

13. $\mathrm{Xu}, \mathrm{Z}$.S. Uncertain linguistic aggregation operators based approach to multiple attribute group decision making under uncertain linguistic environment. Inf. Sci. 2004, 168, 171-184. [CrossRef]

14. Xu, Z.S. Induced uncertain linguistic OWA operators applied to group decision making. Inf. Fusion 2006, 7, 231-238. [CrossRef] 
15. Wei, G.W. Uncertain linguistic hybrid geometric mean operator and its application to group decision making under uncertain linguistic environment. Int. J. Uncertain. Fuzz. Knowl.-Based Syst. 2009, 17, 251-267. [CrossRef]

16. Wei, G.W.; Zhao, X.F.; Lin, R.; Wang, H.J. Uncertain linguistic Bonferroni mean operators and their application to multiple attribute decision making. Appl. Math. Model. 2013, 37, 5277-5285. [CrossRef]

17. Zhang, H. Uncertain linguistic power geometric operators and their use in multiattribute group decision making. Math. Probl. Eng. 2015, 2015, 948380. [CrossRef]

18. Jun, Y.B.; Kim, C.S.; Yang, K.O. Cubic sets. Ann. Fuzzy Math. Inform. 2012, 4, 83-98.

19. Ye, J. Multiple attribute decision-making method based on linguistic cubic variables. J. Intell. Fuzzy Syst. 2018, 34, 2351-2361. [CrossRef]

20. Lu, X.P.; Ye, J. Dombi aggregation operators of linguistic cubic variables for multiple attribute decision making. Information 2018, 9, 188. [CrossRef]

21. Ye, J. Aggregation operators of neutrosophic linguistic numbers for multiple attribute group decision making. SpringerPlus 2016, 5, 1691. [CrossRef] [PubMed]

22. Cui, W.H.; Ye, J.; Shi, L.L. Linguistic neutrosophic uncertain numbers and their multiple attribute group decision-making method. J. Intell. Fuzzy Syst. 2018. [CrossRef]

23. Fang, Z.B.; Ye, J. Multiple attribute group decision-making method based on linguistic neutrosophic numbers. Symmetry 2017, 9, 111. [CrossRef]

24. Fan, C.X.; Ye, J.; Hu, K.L.; Fan, E. Bonferroni mean operators of linguistic neutrosophic numbers and their multiple attribute group decision-making methods. Information 2017, 8, 107. [CrossRef]

25. Wang, Y.M.; Liu, P.D. Linguistic neutrosophic generalized partitioned Bonferroni mean operators and their application to multi-attribute group decision making. Syemmtry 2018, 10, 160. [CrossRef]

26. Shi, L.L.; Ye, J. Cosine measures of linguistic neutrosophic numbers and their application in multiple attribute group decision-making. Information 2017, 8, 117.

27. Shi, L.L.; Ye, J. Multiple attribute group decision-making method using correlation coefficients between linguistic neutrosophic numbers. J. Intell. Fuzzy Syst. 2018, 35, 917-925. [CrossRef]

28. Ye, J. Linguistic neutrosophic cubic numbers and their multiple attribute decision-making method. Information 2017, 8, 110. [CrossRef]

29. Fan, C.X.; Ye, J. Heronian mean operator of linguistic neutrosophic cubic numbers and their multiple attribute decision-making methods. Math. Probl. Eng. 2018, 2018, 4158264. [CrossRef]

30. Ye, J.; Cui, W.H. Operations and aggregation methods of single-valued linguistic neutrosophic interval linguistic numbers and their decision making method. Information 2018, 9, 196. [CrossRef]

31. Ye, J. Multiple attribute decision-making methods based on expected value and similarity measure of hesitant neutrosophic linguistic numbers. Cogn. Comput. 2018, 10, 454-463. [CrossRef]

32. Cui, W.H.; Ye, J. Multiple attribute decision-making method using similarity measures of hesitant linguistic neutrosophic numbers regarding least common multiple cardinality. Symmetry 2018, 10, 330. [CrossRef]

(C) 2018 by the authors. Licensee MDPI, Basel, Switzerland. This article is an open access article distributed under the terms and conditions of the Creative Commons Attribution (CC BY) license (http://creativecommons.org/licenses/by/4.0/). 